\title{
Effects of institutional volumes on operative outcomes for aortic root replacement in North America
}

\author{
G. Chad Hughes, MD, Yue Zhao, PhD, J. Scott Rankin, MD, John E. Scarborough, MD, Sean O’Brien, PhD, \\ Joseph E. Bavaria, MD, Walter G. Wolfe, MD, Jeffrey G. Gaca, MD, James S. Gammie, MD, \\ David M. Shahian, MD, and Peter K. Smith, MD
}

\begin{abstract}
Objectives: Hospital procedure volume has been strongly associated with postoperative mortality for a number of complex cardiovascular procedures. Although not yet described, a similar relationship might be expected for surgical procedures involving the aortic root and/or ascending aorta. The present study sought to evaluate the relationship between the volume of aortic root replacement procedures and the operative results for centers in North America.
\end{abstract}

\begin{abstract}
Methods: Patient-level data for 13,358 elective aortic root and aortic valve-ascending aortic procedures performed from 2004 through 2007 were obtained from 741 North American hospitals participating in the Society of Thoracic Surgeons Adult Cardiac Surgery Database. Marginal logistic regression modeling was used for risk adjustment. The hospital procedure volume was the primary predictor variable. Patient demographics, comorbid conditions, and operative characteristics were included as the predictor variables for risk adjustment. The primary outcome measures included unadjusted operative mortality and adjusted odds ratio for mortality.
\end{abstract}

Results: The preoperative patient risk profiles were similar at all center volume levels, and the overall unadjusted operative mortality was $4.5 \%$. The unadjusted operative mortality increased with decreasing case volume, from $3.4 \%$ in the highest volume centers to $5.8 \%$ in the lowest volume centers. Whether hospital volume was assessed as a categorical or continuous variable, its relationship with the adjusted odds ratio for mortality was nonlinear. A negative association was seen between the hospital procedural volume and adjusted odds ratio for mortality $(P<.001)$ that was most pronounced among hospitals performing fewer than 30 to 40 procedures annually.

Conclusions: Patients undergoing elective aortic root or combined aortic valve-ascending aortic surgery at North American hospitals that performed fewer than 30 to 40 of such procedures annually have greater riskadjusted mortality than those undergoing surgery in higher volume hospitals. Causative factors for this inverse association between hospital volume and mortality deserve additional analysis. (J Thorac Cardiovasc Surg 2013;145:166-70)

Hospital procedure volume has been shown to be strongly associated with postoperative mortality for a number of complex cardiovascular and thoracic surgical procedures, including esophagectomy, ${ }^{1}$ lung cancer resection, ${ }^{2}$ open descending thoracic aortic aneurysm repair, ${ }^{3}$ and both open and endovascular abdominal aortic aneurysm repair. ${ }^{4}$ Although not yet described, a similar hospital volumeoutcomes relationship might be expected for surgical procedures involving the aortic root and/or ascending aorta. Perhaps even more so than with other cardiothoracic procedures, operations involving the aortic root require a high

From the Society of Thoracic Surgeons Database, Durham, NC.

Disclosures: Authors have nothing to disclose with regard to commercial support.

Presented in part at the 2010 American Heart Association Scientific Sessions, Chicago, Illinois, November 13-17, 2010.

Received for publication Aug 28, 2011; accepted for publication Oct 4, 2011; available ahead of print Feb 3, 2012.

Address for reprints: G. Chad Hughes, MD, Division of Thoracic and Cardiovascular Surgery, Department of Surgery, Duke University Medical Center, DUMC Box 3051, Durham, NC 27710 (E-mail: gchad.hughes@duke.edu).

$0022-5223 / \$ 36.00$

Copyright (c) 2013 by The American Association for Thoracic Surgery doi:10.1016/j.jtcvs.2011.10.094 degree of complex peri- and postoperative care. This complexity is reflected in the results of a recent survey analysis of the Society of Thoracic Surgeons (STS) Adult Cardiac Surgery Database (ACSD) showing an approximately $10 \%$ overall mortality rate after aortic root or simultaneous aortic valve and ascending aortic surgery, ${ }^{5}$ a proportion considerably greater than previously published reports from high-volume thoracic aortic centers. ${ }^{6-9}$

Thus, the purpose of the present study was to determine the relationship between the hospital procedure volume and postoperative outcomes after elective aortic root surgery for centers in North America using the STS ACSD. We hypothesized that patients undergoing elective aortic root procedures at higher volume centers would achieve superior risk-adjusted postoperative outcomes compared with patients undergoing these procedures at lower volume centers.

\section{METHODS}

The STS ACSD was established in 1989 to report surgical outcomes after cardiothoracic surgical procedures. ${ }^{10}$ The sites enter patient data using uniform definitions (available at: http://www.sts.org) and certified 


\section{Abbreviations and Acronyms \\ ACSD $=$ Adult Cardiac Surgery Database \\ NIS = Nationwide Inpatient Sample \\ STS $=$ Society of Thoracic Surgeons}

software systems. Although participation in the STS ACSD is voluntary, data completeness is high, with overall preoperative risk factors missing for fewer than $5 \%$ of submitted cases. At present, more than $90 \%$ of North American cardiac surgery centers submit data. The accuracy of submitted cases has been confirmed in an independent comparison of the hospital coronary artery bypass graft surgery volume and mortality rates reported to the STS ACSD versus those reported to the Centers for Medicare and Medicaid Services. ${ }^{11}$ In addition, database accuracy is ensured annually using randomly selected on-site audits.

For the present study, patients who underwent either aortic root replacement or combined aortic valve replacement with simultaneous ascending aortic replacement in 741 hospitals participating in the STS ACSD from 2004 to 2007 were included. Because of measured similarities in patient baseline characteristics and raw unadjusted mortality, together with uncertainties regarding the exact procedural details in the data set, all categories of patients involving aortic root replacement or aortic valve plus ascending aortic replacement were analyzed as 1 population. Aortic dissection and nonelective cases were excluded; thus, the final analysis included patient-level data for 13,358 elective aortic root/aortic valve-ascending aortic procedures.

The descriptive statistics of the patient characteristics and outcomes are presented as group mean values for continuous variables and frequencies or percentages for the categorical variables. The Wilcoxon rank sum test was used to compare the distribution of continuous variables between groups, and the Mantel-Haenszel test was used for categorical variable comparisons. The primary outcome variable was operative mortality, defined as death from any cause, either in-hospital or within 30 days of the index thoracic aortic operation. The average annual hospital volumes were determined from the operations submitted to the STS ACSD from January 2004 to December 2007 (the period when data collection form version 2.52 was used). The average annual volume for each hospital was calculated as the number of procedures during the study period divided by the number of months that the hospital participated in the database during the study period multiplied by 12 . The hospital-specific average annual volumes ranged from 0.25 to about 100 cases annually. The association between operative mortality and volume was assessed by managing volume as either a continuous or a categorical variable with 4 categories (group 1, lowest to group 4, highest). The cutpoints $(<6,6-13,13-30$, and $30-100$ cases/yr) were selected to ensure an approximately equal number of patients in each category. The marginal logistic regression model using generalized estimating equations method was used for risk adjustment to account for the correlation among patients within the same participant group. The unadjusted and risk adjusted odds ratios of operative mortality and the corresponding $95 \%$ confidence intervals are reported for each volume category relative to the performance of the hospitals with fewer than 6 cases annually.

To allow for possible nonlinear effects, the volume was also modeled as a continuous variable, using restricted cubic splines with knots at 3 (10th percentile), 13 (50th percentile), and 63 (90th percentile) cases annually. Sensitivity to the number of knots and the choice of knot locations was assessed by refitting the logistic models with the knots at various percentiles of the empirical distribution of hospital volume. The shape of the estimated volume-outcome association was generally consistent across these different model specifications. The risk-adjusted results, determined from the model with smaller Akaike's Information Criteria, are presented. The risk-adjusted models included the following covariates: age, left ventricular ejection fraction, body surface area, serum creatinine, time trend, active endocarditis, need for dialysis, atrial fibrillation, female gender, hypertension, immunosuppressive treatment, presence of an intraaortic balloon pump, inotrope use, peripheral vascular disease, unstable angina (no myocardial infarction $<7$ days), left main disease, aortic stenosis, aortic insufficiency, mitral stenosis, mitral insufficiency, tricuspid insufficiency, chronic lung disease, cerebrovascular disease or cerebrovascular accident, diabetes, number of diseased coronary vessels, myocardial infarction, race, admission status, congestive heart failure, New York Heart Association class, reoperation, and concomitant coronary artery bypass grafting. The use of circulatory arrest during a procedure was not available as a coding variable on the data collection form version 2.52 used for the present analysis. The coding details for these variables have been previously described. ${ }^{12}$ All analyses were performed using SAS, version 8.2, software (SAS Institute, Cary, NC).

\section{RESULTS}

Of the 741 centers included in the present study, $72 \%$ $(\mathrm{n}=534)$ were in the lowest volume category $(<6$ cases/yr), and $3 \%(n=22)$ performed the highest volume $(30$ 100 cases/yr). Thus, $25 \%$ of the total cases ( $n=3404$ of 13,358 ) were performed at the $3 \%$ of centers in the highest volume category. The preoperative patient risk profiles were minimally different between low- and high-volume centers (Table 1). Two major exceptions to this finding were the number of patients who had undergone previous cardiac surgery (eg, coronary artery bypass graft surgery, valve, other) and the number with endocarditis, with both groups more likely to undergo surgery in the higher volume hospitals.

The overall unadjusted 30-day/in-hospital mortality rate for all 13,358 patients in the study was $4.48 \%$ and the overall stroke rate was $2.25 \%$. The rates of other major complications for the entire cohort included the need for reoperation $(11.37 \%)$, perioperative myocardial infarction $(2.27 \%)$, prolonged ventilation $(14.49 \%)$, and renal failure $(5.27 \%)$. The mean postprocedural length of stay was 8.6 days (interquartile range $5.0-9.0$ ).

An increasing institutional case volume was associated with lower unadjusted and risk-adjusted mortality $(P<.001$; Table 2). Patients undergoing aortic root or combined aortic valve plus ascending aortic replacement were $58 \%$ less likely to experience operative mortality when undergoing surgery in a highest volume versus lowest volume center. When volume was assessed as a continuous variable, the relationship was nonlinear, with a significant negative association between risk-adjusted mortality and procedural volume observed in the lower volume range (procedural volumes $<30-40$ cases/year; Figure 1). The most common cause of 30-day/in-hospital mortality was cardiac and did not vary significantly between the lower and higher volume centers (Table 3 ).

The unadjusted rates of major morbidity stratified by center procedural volume are presented in Table 4. Cardiopulmonary bypass and aortic crossclamp times averaged 181 minutes (interquartile range, 130-215) and 134 minutes 
TABLE 1. Patient demographics

\begin{tabular}{|c|c|c|c|c|c|c|}
\hline \multirow[b]{2}{*}{ Variable } & \multirow[b]{2}{*}{ Overall group $(n=13,358)$} & \multicolumn{4}{|c|}{ Hospital volume } & \multirow[b]{2}{*}{$P$ value* } \\
\hline & & $<6(n=3331)$ & 6-13 $(n=3308)$ & 13-30 $(n=3315)$ & $30-100(n=3404)$ & \\
\hline Hospital number & 741 & 534 & 126 & 59 & 22 & - \\
\hline Mean age (yr) & 60.23 & 60.96 & 60.56 & 59.45 & 59.98 & .0005 \\
\hline Female gender & 30.19 & 30.95 & 30.68 & 29.92 & 29.23 & .0941 \\
\hline Mean BSA $\left(\mathrm{m}^{2}\right)$ & 1.99 & 1.99 & 1.99 & 1.99 & 1.99 & .2285 \\
\hline Diabetes & 12.43 & 12.64 & 13.18 & 12.40 & 11.52 & .0970 \\
\hline Hypertension & 65.94 & 68.33 & 67.44 & 63.71 & 64.31 & $<.0001$ \\
\hline Dyslipidemia & 49.54 & 47.67 & 49.27 & 46.40 & 54.67 & $<.0001$ \\
\hline Smoker & 50.99 & 52.78 & 51.03 & 49.95 & 50.21 & .0240 \\
\hline Chronic lung disease & 16.99 & 19.48 & 17.59 & 16.35 & 14.60 & $<.0001$ \\
\hline PVD & 14.91 & 13.51 & 16.66 & 13.27 & 16.19 & .0888 \\
\hline CVD & 9.93 & 8.65 & 9.28 & 10.26 & 11.52 & $<.0001$ \\
\hline Mean preoperative creatinine & 1.10 & 1.08 & 1.08 & 1.10 & 1.11 & .0003 \\
\hline Endocarditis & 4.27 & 2.82 & 3.63 & 4.77 & 5.82 & $<.0001$ \\
\hline $\mathrm{CAD}$ & 32.73 & 31.82 & 31.26 & 29.77 & 37.93 & $<.0001$ \\
\hline Previous CABG & 4.55 & 3.51 & 3.99 & 5.10 & 5.58 & $<.0001$ \\
\hline Previous valve surgery & 11.13 & 7.93 & 9.46 & 12.79 & 14.28 & $<.0001$ \\
\hline Previous other cardiac surgery & 5.55 & 4.29 & 4.32 & 5.52 & 7.99 & $<.0001$ \\
\hline NYHA class III-IV & 33.71 & 36.17 & 36.49 & 33.93 & 28.37 & $<.0001$ \\
\hline Mean EF $(\%)$ & 54.57 & 54.03 & 54.11 & 54.08 & 55.91 & $<.0001$ \\
\hline
\end{tabular}

$B S A$, Body surface area; $H T N$, hypertension; $P V D$, peripheral vascular disease; $C V D$, cerebrovascular disease; $C A D$, coronary artery disease; $C A B G$, coronary artery bypass grafting; $N Y H A$, New York Heart Association; $E F$, ejection fraction. *Test for any difference among 4 hospital volume groups.

(interquartile range, 96-162), respectively, for the entire cohort, with no meaningful differences between the lowand high-volume centers. Patients treated at the higher volume centers were less likely to require blood products $(47 \%$ vs $57 \% ; P<.0001)$ and spent less time in the intensive care unit $(82.8$ vs 93.1 hours; $P<.0001)$ compared with those treated at lowest volume centers. The postprocedural length of stay did not vary significantly between the groups.

\section{DISCUSSION}

In the present analysis of the STS ACSD, a clear inverse association between hospital procedure volume and postoperative mortality was observed after elective aortic root and aortic valve/ascending aortic surgery. This inverse association between hospital volume and outcomes appeared most pronounced among centers that performed fewer than 30 to 40 elective aortic root procedures annually. Evidence for this volume-outcome relationship persisted after adjustment for various patient-level factors and using statistical techniques that accounted for the potential clustering of outcomes within specific hospitals. Furthermore, the inverse association was apparent regardless of whether the primary predictor variable, hospital procedure volume, was considered as a trichotomous ordered categorical variable or a continuous variable.

These results are consistent with the findings of previous studies that examined the volume-outcome relationship for thoracic aortic surgery. Cowan and colleagues ${ }^{13}$ used the Nationwide Inpatient Sample (NIS) to demonstrate a near doubling of in-hospital mortality among patients undergoing intact thoracoabdominal aortic aneurysm repair at low-volume hospitals (median volume 1 procedure/yr) compared with patients undergoing the procedure at highvolume hospitals (median volume 12 procedures/yr; $27 \%$ vs $15 \%$ mortality; $P<.001$ ). Similar results were obtained by Schermerhorn and associates ${ }^{3}$ in their NIS-based analysis of intact and ruptured open descending thoracic

TABLE 2. Perioperative mortality stratified by center procedural volume

\begin{tabular}{lccccc}
\hline Variable & Group 1 & Group 2 & Group 3 & Group 4 & P value* \\
\hline Cases/yr & $<6$ & $6-13$ & $13-30$ & $30-100$ & - \\
Centers (n) & 534 & 126 & 59 & 3315 & 3404 \\
Patients (n) & 3331 & 3308 & 3.98 & 3.35 & - \\
UOM & 5.82 & $0.82(0.65-1.02)$ & $0.67(0.52-0.87)$ & $0.56(0.40-0.79)$ & $<.001$ \\
UORM & 1.00 (reference) & $0.79(0.62-1.00)$ & $0.55(0.42-0.73)$ & $0.42(0.31-0.58)$ \\
AORM & 1.00 (reference) & & $<.001$ \\
\hline
\end{tabular}

Data presented as odds ratios, with $95 \%$ confidence intervals in parentheses. UOM, Unadjusted operative mortality; UORM, unadjusted odds ratio for mortality; AORM, adjusted odds ratio for mortality. *Test for any difference among 4 hospital volume groups. 

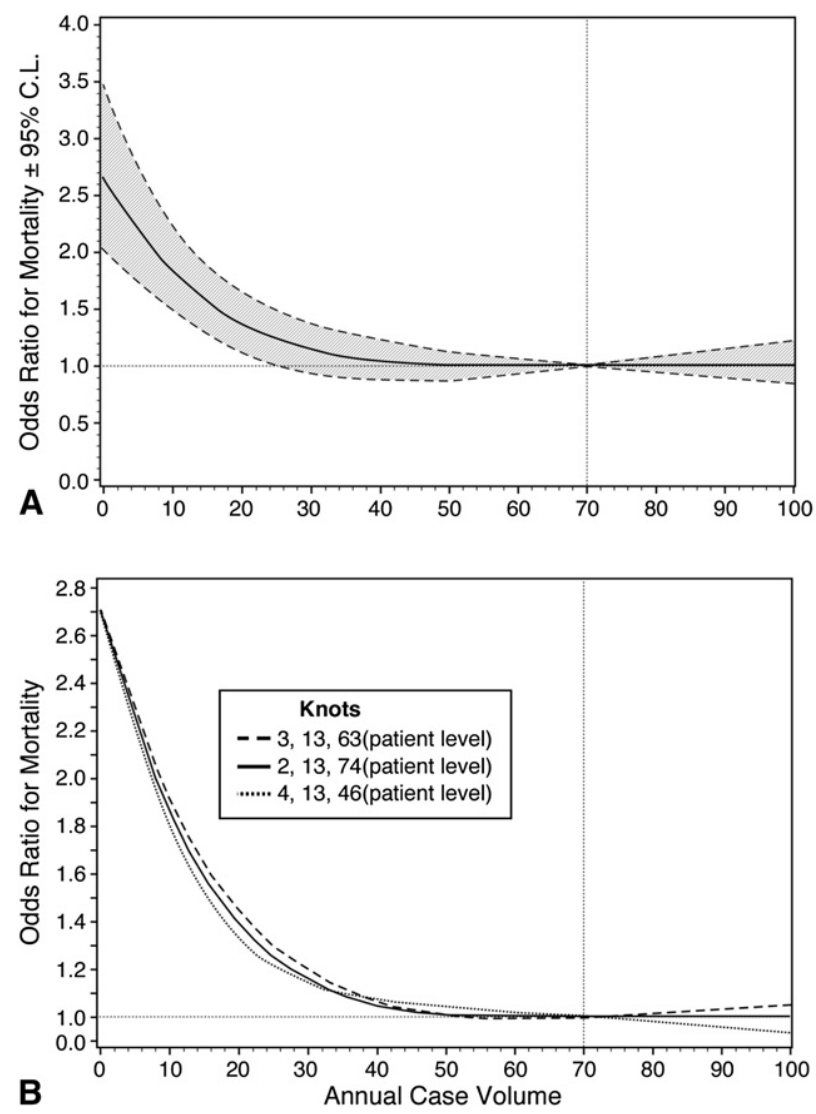

FIGURE 1. A, Observed relationship between annual institutional case volume and risk-adjusted odds ratio for operative mortality \pm 2 standard deviation as assessed with regression analysis. Mortality increased as institutional case volume declined. B, Same relationship as in Figure A, except curve fitting was with spline function with 3 knots. Variation in location of knots had little effect on the relationship.

aneurysm repair. Finally, Knipp and colleagues ${ }^{14}$ used the NIS to assess the hospital volume-outcomes relationship for acute type A aortic dissection repair in the United States. They also found a significant inverse correlation between hospital procedural volume and mortality (34\% in low- volume hospitals vs $25 \%$ in high-volume hospitals, $P=.003$ ) for patients undergoing urgent or emergent repair of acute type A aortic dissection.

The present analysis differed from previously published volume-outcome studies of thoracic aortic surgery in several important ways. First, our study used a prospectively maintained, cardiothoracic surgery-specific clinical database that includes risk-adjustment variables immediately relevant to the outcomes after thoracic aortic surgery (eg, New York Heart Association heart failure classification). In contrast, the aforementioned NIS-based analyses relied on less granular comorbidity data and were subject to the limitations inherent in the use of an administrative discharge-based data source. Second, previous studies considered the hospital volume only as a categorical variable. The present study, however, included 2 models, 1 of which considered hospital volume as a continuous variable. This latter analysis avoided the potential problems accompanying the somewhat arbitrary nature by which hospitals are separated into distinct procedural volume categories. ${ }^{15}$ Finally, the statistical method used in the present study was designed to adjust for potentially important, but unspecified, hospital-related variables other than procedure volume that might result in a clustering of adverse patient outcomes within specific hospitals.

An important limitation of the present analysis was an inability to adjust for individual surgeon volume. The STS database does not provide surgeon-specific data; therefore, the potential effect of individual surgeon procedure volume on the observed hospital-level volume-outcomes relationship could not be determined. As such, we cannot exclude the possibility that a high-volume surgeon who operates within a low-volume hospital might achieve outcomes comparable to those of a low- or even high-volume surgeon who operates within a high-volume hospital.

Nevertheless, the results of the present analysis support the presence of a strong inverse association between hospital volume and postoperative mortality after elective aortic root surgery, especially among hospitals that perform fewer

TABLE 3. Cause of 30-day/in-hospital mortality stratified by center procedural volume*

\begin{tabular}{|c|c|c|c|c|c|}
\hline \multirow[b]{2}{*}{ Primary cause of death } & \multirow[b]{2}{*}{ Overall group } & \multicolumn{4}{|c|}{ Hospital volume } \\
\hline & & $<6$ & 6-13 & 13-30 & $30-100$ \\
\hline Cardiac & 55.09 & 53.61 & 54.09 & 60.61 & 52.63 \\
\hline Pulmonary & 9.85 & 9.79 & 8.18 & 10.61 & 11.40 \\
\hline Neurologic & 8.01 & 7.73 & 8.81 & 6.82 & 8.77 \\
\hline Infection & 7.51 & 6.70 & 7.55 & 6.82 & 9.65 \\
\hline Vascular & 3.67 & 5.15 & 5.03 & 0.00 & 3.51 \\
\hline Renal & 1.34 & 1.55 & 1.89 & 0.76 & 0.88 \\
\hline Valvular & 1.17 & 0.00 & 1.89 & 3.03 & 0.00 \\
\hline Unknown & 2.00 & 2.06 & 2.52 & 0.76 & 2.63 \\
\hline Other & 9.18 & 10.31 & 7.55 & 9.85 & 8.77 \\
\hline Missing & 2.17 & 3.09 & 2.52 & 0.76 & 1.75 \\
\hline
\end{tabular}

Data presented as percentages. ${ }^{*} P=.7787$ for cause of mortality among groups. 
TABLE 4. Perioperative major morbidity stratified by center procedural volume

\begin{tabular}{|c|c|c|c|c|c|c|}
\hline \multirow[b]{2}{*}{ Postoperative complication } & \multirow[b]{2}{*}{ Overall group $(n=13,358)$} & \multicolumn{4}{|c|}{ Hospital volume } & \multirow[b]{2}{*}{$P$ value } \\
\hline & & $<\mathbf{6}(\mathbf{n}=\mathbf{3 3 3 1})$ & 6-13 $(n=3308)$ & 13-30 $(n=3315)$ & $30-100(n=3404)$ & \\
\hline Stroke & 2.25 & 2.34 & 2.39 & 2.32 & 1.97 & .2782 \\
\hline Reoperation & 11.37 & 12.88 & 11.15 & 11.22 & 10.25 & $<.0001$ \\
\hline Prolonged ventilation & 14.49 & 15.82 & 14.18 & 14.09 & 13.87 & .0232 \\
\hline Renal failure & 5.27 & 5.73 & 5.20 & 5.61 & 4.55 & .0745 \\
\hline
\end{tabular}

Data presented as percentages.

than 30 to 40 such procedures annually. Knowledge of this relationship might be helpful in designing policies aimed at improving the overall quality of care provided to patients who require aortic root procedures. One potential policy approach applied to numerous other complex surgical procedures is to use minimum annual aortic root procedure volume requirements as a criterion in defining "Centers of Excellence" in thoracic aortic surgery. ${ }^{16,17}$ However, this approach is subject not only to political obstacles but is also likely untenable from a practical standpoint given geographic factors and the small number of high-volume centers that exist (only $3 \%$ of all North American centers). An alternative and more viable approach is to identify the specific practice standards and processes of care that underlie the superior outcomes achieved by high-volume centers. This information can be disseminated to low-volume centers through the current framework of national meetings and educational programs or through more structured quality improvement initiatives such as exist for solid organ transplantation centers. ${ }^{18}$ Once identified, these same processes of care can also be used as benchmarks to assess the quality of care provided by hospitals where complex thoracic aortic procedures such as aortic root replacement are performed. ${ }^{18}$ Regardless of which policy approach is ultimately deemed most acceptable, the findings of the present analysis strongly suggest that hospital procedure volume currently serves as an important marker of the quality of care provided to patients undergoing aortic root surgery.

\section{References}

1. Allareddy V, Allareddy V, Konety BR. Specificity of procedure volume and in-hospital mortality association. Ann Surg. 2007;246:135-9.

2. Cheung MC, Hamilton K, Sherman R, Byrne MM, Nguyen DM, Franceschi D, et al. Impact of teaching facility status and high-volume centers on outcomes for lung cancer resection: an examination of 13,469 surgical patients. Ann Surg Oncol. 2009;16:3-13.
3. Schermerhorn ML, Giles KA, Hamdan AD, Dalhberg SE, Hagberg R, Pomposelli F. Population-based outcomes of open descending thoracic aortic aneurysm repair. J Vasc Surg. 2008;48:821-7.

4. Brooke BS, Perler BA, Dominici F, et al. Reduction of in-hospital mortality among California hospitals meeting Leapfrog evidence-based standards for abdominal aortic aneurysm repair. J Vasc Surg. 2008;47:1155-64.

5. Rankin JS, Hammill BG, O'Brien SM, Edwards FH, Peterson ED, DeLong ER, et al. Determinants of operative mortality in valvular heart surgery. $J$ Thorac Cardiovasc Surg. 2006;131:547-57.

6. Lima B, Hughes GC, Lemaire A, Jaggers J, Glower DD, Wolfe WG. Short- and intermediate-term outcomes of aortic root replacement with St. Jude mechanical conduits and aortic allografts. Ann Thorac Surg. 2006;82:579-85.

7. Dapunt OE, Easo J, Holzl PP, Murin P, Sudkamp M, Horst M, et al. Stentless full root bioprosthesis in surgery for complex aortic valve-ascending aortic disease: a single center experience of over 300 patients. Eur J Cardiothorac Surg. 2008;33:554-9.

8. Kindo M, Billaud P, Gerelli S, Levy F, Mazzucotelli JP, Eisenmann B. Twentyseven-year experience with composite valve graft replacement of the aortic root. J Heart Valve Dis. 2007;16:370-7.

9. Etz CD, Homann TM, Rane N, Bodian CA, DiLuozzo G, Plestis KA, et al. Aortic root reconstruction with a bioprosthetic valved conduit: a consecutive series of 275 procedures. J Thorac Cardiovasc Surg. 2007;133:1455-63.

10. Ferguson TB, Dziuban SW, Edwards FH, Eiken MC, Shroyer AL, Pairolero PC, et al. The STS National Database: current changes and challenges for the new millennium. Ann Thorac Surg. 2000;69:680-91.

11. Welke KF, Ferguson TB, Coombs LB, Dokholyan RS, Murray CJ, Schrader MA, et al. Validity of the Society of Thoracic Surgeons National Adult Cardiac Surgery Database. Ann Thorac Surg. 2004;77:1137-9.

12. O'Brien SM, Shahian DM, Filardo G, Ferraris VA, Haan CK, Rich JB, et al. The Society of Thoracic Surgeons 2008 cardiac surgery risk models: part 2-isolated valve surgery. Ann Thorac Surg. 2009;88:S23-42.

13. Cowan JA Jr, Dimick JB, Henke PK, Huber TS, Stanley JC, Upchurch GR Jr. Surgical treatment of intact thoracoabdominal aortic aneurysms in the United States: hospital and surgeon volume-related outcomes. J Vasc Surg. 2003;37: 1169-74.

14. Knipp BS, Deeb M, Prager RL, Williams CY, Upchurch GR Jr, Patel HJ. A contemporary analysis of outcomes for operative repair of type A aortic dissection in the United States. Surgery. 2007;142:524-8.

15. Livingston EH, Cao J. Procedure volume as a predictor of surgical outcomes (editorial). JAMA. 2010;304:95-7.

16. Birkmeyer JD, Dimick JB. Potential benefits of the 2003 Leapfrog Standards: effect of process and outcome measures. Surgery. 2004;135:569-75.

17. CMS bariatric surgery national coverage determination. Available from: http:// www.cms.gov/manuals/downloads/ncd103c1_Part2.pdf. Accessed November $22,2010$.

18. Goshima KR, Mills JR Sr, Awari K, Pike SL, Hughes JD. Measure what matters: Institutional outcome data are superior to the use of surrogate markers to define "center of excellence" for abdominal aortic aneurysm repair. Ann Vasc Surg. 2008;22:328-34. 